\title{
Is It Time to Give Athletes a Voice in the Dissemination Strategies of Concussion-Related Information? Exploratory Examination of
} 2444 Adolescent Athletes

\author{
Mark Beakey \\ Technological University Dublin \\ Brian Keenan \\ Technological University Dublin \\ Stephen Tiernan \\ Technological University Dublin, stephen.tiernan@tudublin.ie
}

See next page for additional authors

Follow this and additional works at: https://arrow.tudublin.ie/ittengoth

Part of the Biomedical Engineering and Bioengineering Commons

\footnotetext{
Recommended Citation

This Article is brought to you for free and open access by the School of Engineering at ARROW@TU Dublin. It has been accepted for inclusion in Other Resources by an authorized administrator of ARROW@TU Dublin. For more information, please contact arrow.admin@tudublin.ie, aisling.coyne@tudublin.ie,gerard.connolly@tudublin.ie.
}

Beakey M, Keenan B, Tiernan S, Collins K. Is It Time to Give Athletes a Voice in the Dissemination Strategies of Concussion-Related Information? Exploratory Examination of 2444 Adolescent Athletes. Clin J Sport Med. 2020 Nov;30(6):562-567. doi: 10.1097/JSM.0000000000000653. PMID: 30119086.

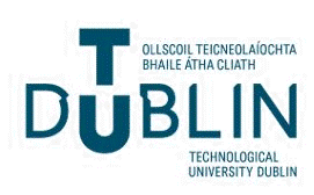


Authors

Mark Beakey, Brian Keenan, Stephen Tiernan, and Kieran Collins

This article is available at ARROW@TU Dublin: https://arrow.tudublin.ie/ittengoth/6 
Concussion Education: Is it time to give athletes a voice in the dissemination strategies of concussion-related information?

Examination of 2444 adolescent athletes

Mark Beakey $^{1}$, Brian Keenan $^{1}$, Stephen Tiernan $^{2}, \&$ Kieran Collins ${ }^{1,3}$

1. Department of Science, Institute of Techno12logy, Tallaght, Dublin, Ireland.

2. Department of Engineering, Institute of Technology, Tallaght, Dublin, Ireland.

3. Gaelic Research Sport Centre, Institute of Technology, Tallaght, Dublin, Ireland.

Corresponding Author:

Mark Beakey, MSc, Department of Science, Institute of Technology, Tallaght, Dublin 24, D24 FKT9, Ireland. Email address- mark.beakey@ postgrad.ittdublin.ie

Abstract Word Count- 245

Word Count- 2883

Number of Tables- 2

Number of Figures- 2

Number of Supplementary Files- 1

Key words: sport related concussion (SRC); adolescent athletes; knowledge translation; gender; educational intervention. 


\begin{abstract}
Background: Translating knowledge from clinical research into practice is a vital step in effectively educating athletes on sport-related concussion. Currently, no research has investigated the opinions of adolescent athletes on the content and delivery methods employed in concussion educational interventions.
\end{abstract}

Objectives: To screen male and female adolescent athletes on their concussion educational histories and future preferred future educational interests in terms of messenger, modality and concussion-related areas of interest.

Methods: A brief cross-sectional survey instrument was delivered to athletes in secondary schools $(n=10)$ and sports clubs $(n=31)$ under supervision of the principal investigator.

Results: 2444 adolescent athletes $($ male $=1854$, female $=590)$ completed the survey. $19.7 \%$ $(n=482)$ of the sample received education in the last 12 months. Male athletes had a significantly higher rate of previous education than female athletes. The methods utilised in previous educational interventions are not matching the interests of the athletes. Gender played a significant role in the preferred educational methods with male and female athletes having significant differences in their choice of educational messenger, modality and areas of interest.

Conclusion: Male and female athletes differ significantly in their favoured educational methods and areas of interests. Future interventions may consider tailoring their knowledge translation strategies to match the specific needs of the stakeholder. As athletes' desire concussion education from a multitude of sources and individuals, it is imperative these sources of information and educational messengers are providing consistent and evidence-based information on sport-related concussion. 


\section{Introduction}

Concussion has become a major public health priority in recent years resulting in an abundance of published research in the medical literature. ${ }^{1}$ The substantial level of research in the area is predicated on sport-related concussion (SRC) being a significant threat to the quality of life of athletes of all ages, genders and levels of play. ${ }^{2}$ Although, due to current scientific limitations, the understanding of this topic remains in its infancy. A universal standardised definition of SRC remains elusive. ${ }^{3}$ The scientific community remain divided on several key aspects of SRC including; identification methods, ${ }^{4}$ management and return-to-play protocols, ${ }^{5}$ recovery diagnoses ${ }^{6}$ and the potential links between repeated concussive and sub-concussive blows with the onset of early neurodegenerative diseases. ${ }^{7}$ However, despite divergent opinions, there is an across-the-board acceptance of SRC being a dangerous brain injury and the need to reduce its prevalence and to manage the injury safely is indubitable. ${ }^{8} \mathrm{~A}$ concussion can produce debilitating symptomatology which may affect an athlete for a number of weeks and may even remain to the fore over several months in the form of post-concussion syndrome. ${ }^{9}$ Athletes may experience motor, cognitive, behavioural and sleep impairments which hinder everyday activities, academic and athletic performance, and can leave the athlete in a compromised emotional state. ${ }^{8}$

The prevalence of SRC is higher in contact or collision-based sports than non-contact sporting activities. ${ }^{10}$ As youth athletes appear to be at a heightened risk of concussion and its associated negative effects, the prompt removal from play and management of the injury for athletes in

such sports is crucial. ${ }^{11}$ Currently, there is no objective diagnostical tool available for concussion and thus, many concussions are overlooked and untreated. ${ }^{8}$ There is an onus on the individual athlete to honestly disclose the presence of any potential concussive symptoms to allow a medical assessment to be undertaken. Previous research on youth athletes has 
emphasized several barriers which deter their honest disclosure of potential concussive events. $^{12-14}$

Over the last decade, the education of important stakeholders such as athletes, parents, coaches and medical personnel on the dangers of SRC has become a topical area of research. ${ }^{15}$ The effective transfer of information and guidelines from clinical research into everyday practice is a difficult task. ${ }^{16,17}$ The efficacy of numerous educational strategies to effectively disseminate information have been investigated. ${ }^{18}$ Unfortunately, many past interventions have methodological flaws that need to be taken into consideration when interpreting their findings. The long term impact of such interventions on athlete knowledge and behaviour remains unknown due to the lack of follow up investigations. ${ }^{18}$ As an increase in knowledge does not equate to behaviour change, educational interventions aimed solely at increasing knowledge have been found ineffective at modifying concussion reporting behaviours in athletes. ${ }^{19}$ To achieve desired results, educators must screen athletes on their underlying motivational beliefs which dictate their reporting of SRC. Generalised educational strategies may be ineffective methods of educating athletes about SRC and efforts to design interventions in a population appropriate manner should be explored.$^{20}$ Recently, researchers have experienced early success utilising behavioural models, such as the theory of planned behaviour, to understand and mitigate these negative reporting behaviours. ${ }^{20,21}$ Although, a step forward in the right direction, these teaching techniques, used exclusively, may not reflect an athlete's educational desires. A screening of athlete interests should be undertaken to allow a tailored intervention to be developed. ${ }^{22}$ If athletes are cognitively engaged throughout the knowledge translation process, they have a better chance of retaining a long-term knowledge of the material provided and adhering to the recommended safety guidelines. ${ }^{23,24}$

To date, only two studies have investigated the concussion educational interests of their target stakeholder. Using an online survey, Kroshus et al. ${ }^{22}$ and Sullivan et al. ${ }^{25}$ screened US 
collegiate athletes and Irish GAA (Gaelic Athletic Association) coaches, respectively. No research has been carried out on high school athletes. Therefore, the aim of this study was to screen male and female high school athletes on their concussion educational histories and preferred future educational interests. The study aimed to 1) assess the difference in previous concussion education rates between male and female athletes, 2) examine the impact of gender on educational desires and 3) to test whether methods utilised in previous educational interventions are matching the interests of the athletes in terms of messenger, modality and content.

\section{Methods}

\section{Sample}

Irish secondary schools $(n=45)$ and sports clubs $(n=78)$ were invited, via email to participate in the study between January 2016 and September 2017. Ten secondary schools (22.2\%) and thirty-one sports clubs (39.7\%) agreed to allow their athletes to take part in the research. Athletes were eligible to participate if they were aged 12 to 18 years and currently played a contact or collision-based team sport at an amateur level at the time of testing. The sample was sub-categorised according to the gender of the participants. Prior to testing, the principal investigator visited each school and club to outline the design and purpose of the research. The respective parents or guardians were also informed of the study via letter and email. Participation was voluntary, and the anonymity of the athletes was preserved. No parent or athlete objected to the study and the research was permitted by the Institute of Technology, Tallaght, Dublin's Research and Ethics Committee.

\section{Instrumentation}

A brief cross-sectional survey instrument consisting of a mixture of dichotomous and multiple choice styled questions (9 questions) was administered to the athletes (Supplementary File 1). 
Prior to testing, the survey instrument was reviewed by a panel of three experts to determine content validity. Each question was evaluated for relevancy and needed unanimous approval by the expert panel to be included in the survey. Each question was determined as relevant, yet, the terminology employed in two of the questions was simplified to ensure there was no misunderstanding or confusion among the students. A pilot test was carried out using a randomised sample of fifty athletes $(n=29$ male, $n=21$ female $)$ across three and seven of the participating schools and clubs, respectively. To assess for internal consistency reliability, the pilot sample completed the survey on two occasions with a seven-day period between the first and second round of testing. The survey recorded a Cronbach alpha score of 0.91 and thus, was deemed a reliable measure of an athlete's concussion educational history and future educational desires.

The survey comprised of two sections and took approximately ten minutes to complete. The first section examined the athletes' demographic information. The second section questioned athletes on their concussion educational history and their favoured methods of future SRC education. This section was split into three categories; 1) educational messenger, 2) modality and 3) areas of concussion-related interest.

\section{Procedure}

Under supervision of the principal investigator, one paper survey was administered per athlete in an exam environment. Testing was carried out in groups ranging from 10 to 50 students at the premises of the participating schools and clubs between March 2016 and September 2017. All participating athletes within the same school or club were tested on the same day to limit athlete interaction.

\section{Statistical analysis}


Descriptive statistics were used to calculate the athlete frequency responses in each category of the survey (messenger, modality and areas of interest). The data for each category was split according to gender and educational history, and was examined using separate contingency tables. A Pearson chi-squared test of association was used to compare the proportion of responses for each above variable. An alpha priori level of 0.05 was chosen for the study and all descriptive and inferential statistical analyses were performed using SPSS statistical software version 24 (IBM Corporation, Armonk, NY).

\section{Results}

In total, 2502 athletes undertook the survey instrument, however, 58 athletes failed to complete the survey. Thus, their responses were discarded from the findings leaving 2444 athlete responses $(1854=$ male; female $=590)$ eligible for review. The sample consisted predominantly of rugby players $(44.9 \%, \mathrm{n}=1097)$, followed by soccer $(32.7 \% . \mathrm{n}=800)$ and GAA $(22.4 \%, \mathrm{n}=547)$ players (Gaelic football, hurling and camogie). 35.2\% $(\mathrm{n}=860)$ reported receiving previous education on SRC with $19.7 \%(n=482)$ of athletes receiving some education in the last 12 months. The concussion educational histories of the sample are subcategorised by gender in Table 1 . A comparison between past methods of concussion education received by the athletes and their favoured future methods is displayed in Figure 1 and 2. Male adolescent athletes have received significantly more previous education on SRC than female athletes $\left(41 \%\right.$ vs $\left.17 \% ; \chi^{2}=73.5, \mathrm{p}<0.001\right)$.

$$
\begin{aligned}
& * * * \text { Insert Table } 1 \text { near here } * * * \\
& * * * \text { Insert Table } 2 \text { near here } * * *
\end{aligned}
$$

Gender also played a significant role in the preferred educational methods within the sample $(\chi 2 \geq 546, p<0.001)$. With regards to modality, male athletes were significantly more inclined to opt for an "interactive" (46\% vs $12 \%: \chi 2=13.7, \mathrm{p}<0.001)$ or "on-field demonstration" 
$\left(42 \%\right.$ vs $\left.16 \% ; \chi^{2}=84.3, p<0.001\right)$ compared with female athlete who had a greater desire for an "informational handout/poster" (8\% vs 40\%; $\chi 2=289.2, \mathrm{p}<0.001)$ than males. With reference to educational messenger, male athletes were more likely to choose a "professional/famed coach" (71\% vs 30\%; $\chi 2=254.4, \mathrm{p}<0.001)$ or "professional/famed player" $\left(69 \%\right.$ vs $\left.35 \% ; \chi^{2}=82.5, \mathrm{p}<0.001\right)$ than females. Both male $(45 \%)$ and female $(67 \%)$ athlete sought their coach to be involved in their concussion education. In terms of areas of future learning, female athletes had a considerably greater interest in the potential "long-term complications" (38\% vs 57\%; $\chi 2=37.4, \mathrm{p}<0.001)$ of SRC and "impact on academics" (20\% vs $\left.63 \% ; \chi^{2}=258.7, \mathrm{p}<0.001\right)$ whereas male athletes had considerable more eagerness to understand the "short-term complications" (79\% vs 62\%; $\chi 2=17.1, \mathrm{p}<0.001)$ of SRC and the "impact on athletic performance" (71\% vs 33\%; $\chi 2=102, \mathrm{p}<0.001)$ (Table 2$)$.

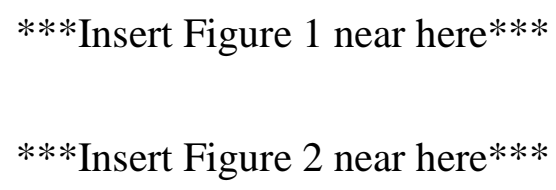

\section{Discussion}

This study sought to examine the concussion educational histories, preferred future educational methods and concussion-related areas of interest in a large cohort of high school athletes. As education is a vital step in improving athlete reporting behaviours, sporting, medical and government bodies are tasked with disseminating information to their respective sporting populations. In the United States for example, education on SRC is mandated for youth athletes under new government legislation and for collegiate athletes through NCAA policy. ${ }^{26}$ It is imperative that the education being delivered to athletes, coaches and parents is matching the specific need of each stakeholder. ${ }^{17}$

\section{Previous education on SRC}


Despite increased emphasis placed on the issue of SRC by sporting organisations and improved media attention, there is still a worrying lack of concussion education in adolescent athletes. High school athletes are more susceptible to SRC and carry an increased risk of a debilitating symptomology than older athletes. ${ }^{27}$ Given that female athletes may be at an even higher risk of suffering a concussion and its associated negative effects, ${ }^{28}$ their significantly lower rate of previous education than their male counterparts (17\% vs $41 \%)$ is troubling and requires urgent intervention by their parents, clubs and schools. Over $80 \%$ of the sample did not receive any education on SRC in the last 12 months indicating the urgent need of sporting organisations, schools and medical bodies to explore effective knowledge translation strategies to disseminate concussion-related education to athletes on a nationwide scale.

\section{Athlete interests in concussion education}

As illustrated in Figure 1, there is a large disconnect between the past methods of concussion education athletes have received and their preferred future methods of education. Most athletes recalled receiving an "informational handout/poster" (50\%) and/or a "general conversation" (51\%) in their previous SRC education despite only a small proportion of the overall sample favouring these modalities. The use of a handout or poster has become common practice in the concussion education of athletes. ${ }^{29}$ However, the efficacy of these passive approaches in modifying behaviour has been questioned. ${ }^{22}$ Currently, the usage of online platforms for concussion education is failing to match the needs of the athletes (Table 2). With the emergence of readily accessible smartphones, $92 \%$ of adolescents' report being online daily with $71 \%$ using more than one social media application. ${ }^{30}$ Research into internet-based applications such as Facebook ${ }^{31}$ and Twitter ${ }^{32}$ have highlighted their potential ability to educate a wide range of adolescent athletes on SRC. To date, many sporting organisations are failing to provide transparent, informative and consistent information on SRC on their respective websites. ${ }^{33} \mathrm{~A}$ recent review of concussion related websites also found varying standards of content, delivery 
and readability of information. ${ }^{34}$ Future research into the knowledge transfer of concussionrelated information using social media and other online platforms is warranted. ${ }^{35}$

\section{Gender differences}

Previous research has proposed tailoring concussion education for differing members within a sample population according to certain concussion modifiers including age ${ }^{36}$ concussion history, ${ }^{33}$ and sport ${ }^{10}$. Within the sample, the preferred educational interests of male athletes differ significantly from female athletes. Therefore, it may also be advantageous to modify future interventions according to the gender of the athletes. As displayed in Table 2, male athletes were significantly more likely to choose an "interactive demonstration" (46\% vs 12\%) and/or an "on-field demonstration" (42\% vs 16\%) as their modality of choice than female athletes. It may be postulated that female athletes may not be as comfortable learning in an active sporting atmosphere or engaging in an open dialogue about SRC with an educational messenger.

\section{“On field" learning approach}

Chinn \& Porter $^{37}$ emphasised the potential efficacy of a procedural learning approach to concussion education by educating athletes in a setting representative of a game-day environment. If athletes are taught in a slow-paced environment through a lecture or video for example, their cognitive processes may be ill equipped to access this information during the contrasting demands of a hectic and adrenaline filled game scenario. ${ }^{37}$ "On-field" educational strategies may be an effective, yet vastly underutilised method of educating athletes on SRC (Table 1) and should be explored further in future research. However, if educators seek to use these interactive methods appropriately, on female athletes especially, they must cultivate an inclusive learning atmosphere to allow the athletes to feel comfortable to actively participate during the intervention. 


\section{Educational messenger}

Most female athletes (70\%) seek a "medical professional" as their concussion educational messenger (Table 2). With recent legislature in youth sport, medical professionals have an increased responsibility in the identification and management of SRC. ${ }^{26}$ For instance, statewide US legislation mandates a clinical diagnosis of the injury and written clearance from a medical professional prior to returning to play. ${ }^{38}$ As female athletes also seek medical personnel in their concussion education, it is imperative these personnel, have up-to-date knowledge of SRC, have the confidence to educate athletes and can effectively distribute concussion-related medical information to their lay audience. ${ }^{39}$

In contrast, male athletes are keen to have a "professional/famed player" (71\%) or "professional/famed coach" (69\%) in the educational process (Table 2). The novelty of having an inspirational figure leading their concussion education seems important within the male sample. If these famed figures provide their own insight into the negative complications of underreporting and encourage honest disclosure of concussive symptoms, it may resonate with athletes and facilitate a positive behaviour change. In alignment with previous research on collegiate athletes, ${ }^{22} 50 \%$ of the sample wish for their own coach to be a part of their future concussion education (Table 2). Previous research has also highlighted the influence of the coaches' approval on their athletes' decision-making processes. ${ }^{14,40}$ As coaches have a considerable impact on their players, they have an obligation to promote a positive reporting culture where athletes will not be punished for openly disclosing their injury. ${ }^{41,42}$ Annual educational strategies to improve coach knowledge, attitude and compliance to SRC management guidelines are warranted.

Adolescent athletes are receiving and requesting concussion-related education from multiple sources (Figure 1). It is imperative these sources are disseminating an evidence-based uniform 
message on SRC. The establishment of a state or national taskforce dedicated to concussion education, management and prevention may be necessary to ensure all educational messengers have competent knowledge and are providing a consistent and singular message on SRC to their athletes. ${ }^{33}$ Screening athletes on their knowledge and reporting behaviours using behavioural models are effective methods in tailoring educational interventions for a specific target audience. ${ }^{20,21}$ To achieve enhanced improvements in reporting behaviour however, it may be necessary to also include the athletes' educational desires to guide the implementation and dissemination strategies of a personalised intervention..

\section{Study limitations}

The study employed a single methodological approach utilising a quantitative questionnaire. Future qualitative research of a target population may be used to delve deeper into their rationale and motivation behind their educational interests prior to designing an appropriate intervention. As this was the first study to investigate the concussion educational interests of high school athletes, the application of the findings in future interventions is unclear. Future research evaluating the inclusion of athlete interests in the knowledge translational strategies of concussion interventions is needed.

\section{Conclusion}

When establishing suitable concussion educational interventions, allowing athletes to voice their opinion on which information is disseminated, who delivers it and how they deliver it, may be a simple approach to retain athlete cognitions throughout the educational process. There is a disconnect between previous concussion education received and future education desired amongst high school athletes. Male and female athletes seek further education on SRC however, there is a significant difference in their preferred methods of future education. 
Acknowledgements: The research was supported by the Institute of Technology, Tallaght, Dublin through their President's Research Award Scheme 2016

Competing Interests: None

\section{What is already known about the topic?}

Adolescent athletes who participate in contact or collision-based sport are at a heightened risk of suffering a concussion and its negative associative effects.

Effective knowledge translation strategies are needed to complement existing evidencebased concussion guidelines to enable athletes to uptake information and modify behaviour.

\section{What does this study add?}

There is a large discrepancy between the previous methods of education athletes have received on sport-related concussion and their preferred future methods of education.

$>$ Male and female athlete differ significantly on their educational desires.

$>$ Prior to designing an intervention, the target audience should be allowed voice their opinion on the knowledge translation strategies to ensure the methods incorporated are matching their interests.

\section{Tables and Figures}

Table 1. Athlete Demographics and Educational History

Table 2. Preferred Methods of For Future Concussion Education

Figure 1. Modality used in Previous Intervention versus Desired Future Modality 
Figure 1 Caption: $\uparrow$ Significant difference between previous modality received and future modality desired within the sample $(\mathrm{P}<0.05)$.

Figure 2. Messenger used in Previous Intervention versus Desired Future Messenger

Figure 2 Caption: $\dagger$ Significant difference between previous messenger received and future messenger desired within the sample $(\mathrm{P}<0.05)$.

\section{References}

1. Wiebe DJ, Comstock RD, Nance, ML. Concussion research: a public health priority. Inj. Prev 2011;17:69-70.

2. Broglio SP. et al. National Athletic Trainers' Association Position Statement: Management of Sport Concussion. J. Athl. Train 2014;49:245-65.

3. McCrory P. et al. What is the definition of sports-related concussion : a systematic review. Br. J. Sports Med 2017;51:877-87.

4. McCrea M. et al. Role of advanced neuroimaging, fluid biomarkers and genetic testing in the assessment of sport-related concussion : a systematic review. Br. J. Sports Med 2017;51:919-29.

5. Broglio SP, Collins MW, Williams RM, et al. Current and emerging rehabilitation for concussion: A review of the evidence. Clin. J. Sport Med 2015;34:213-31.

6. Kamins J. et al. What is the physiological time to recovery after concussion? Systematic review. Br J Sport. Med 2017;51:935-40.

7. Iverson GL, Gardner AJ, McCrory P et al. A critical review of chronic traumatic encephalopathy. Neurosci Biobehav Rev 2015;56:276-93. 
8. McCrory, P. et al. Consensus statement on concussion in sport - the $5^{\text {th }}$ international conference on concussion in sport held in Berlin, October 2016. Br. J. Sports Med 2017; bjsports-2017-097699.

9. Manzanero S. et al. Post-concussion recovery in children and adolescents: A narrative review. J. Concussion 2017;1:1-8.

10. Lincoln AE. et al. Trends in concussion incidence in high school sports: a prospective 11-year study. Am. J. Sports Med 2011;39:958-63.

11. Makdissi M. et al. Revisiting the modifiers: how should the evaluation and management of acute concussions differ in specific groups? Br. J. Sports Med $2013 ; 47: 314-20$.

12. McCrea M, Hammeke T, Olsen G et al. Unreported Concussion in High School Football Players: Implications for Prevention. Clin. J. Sport Med 2004;14:13-7.

13. Kroshus E, Kubzansky LD, Goldman RE et al. Norms, Athletic Identity, and Concussion Symptom Under-Reporting Among Male Collegiate Ice Hockey Players: A Prospective Cohort Study. Ann Behav Med 2015;49:95-103.

14. Chrisman SP, Quitiquit C, Rivara FP. Qualitative study of barriers to concussive symptom reporting in high school athletics. J Adolesc Heal 2013;52:330-5.

15. Donaldson A. et al. Translating Guidelines for the Diagnosis and Management of Sports-Related Concussion Into. Am J Lifestyle Med 2014;10:120-35.

16. Finch CF, McCrory P, Ewing MT et al. Concussion guidelines need to move from only expert content to also include implementation and dissemination strategies. Br J Sport Med 2012;bjsports-2012.

17. Kemp JL, Newton JD, White PE et al. Implementation of concussion guidelines in 
community Australian Football and Rugby League-The experiences and challenges faced by coaches and sports trainers. J Sci Med Sport 2016;19:305-10.

18. Caron JG, Bloom GA, Falcão WR et al. An examination of concussion education programmes: a scoping review methodology. Inj Prev 2015;21:301-8.

19. Mrazik M. et al. A qualitative review of sports concussion education: prime time for evidence-based knowledge translation. Br J Sports Med. 2015;49:1548-53.

20. Kroshus E, Baugh CM, Daneshvar DH et al. Understanding Concussion Reporting Using a Model Based on the Theory of Planned Behavior. J Adolesc Heal 2014;54:269-74.

21. Register-Mihalik J. et al. Using theory to understand high school aged athletes' intentions to report sport-related concussion: Implications for concussion education initiatives. J Athl Train 2013;48:645-53.

22. Kroshus E. \& Baugh CM. Concussion Education in U.S. Collegiate Sport: What Is Happening and What Do Athletes Want? Heal Educ Behav 2015;1-9.

23. Provvidenza CF. \& Johnston, K. M. Knowledge transfer principles as applied to sport concussion education. Br J Sport Med 2009;43:i68-i75.

24. Provvidenza C. et al. From consensus to action: knowledge transfer, education and influencing policy on sports concussion. Br J Sports Med 2013;47:332-8.

25. Sullivan L. \& Molcho M. What do coaches want to know about sports-related concussion? A needs assessment study. J Sport Heal Sci 2017;1-7.

26. Chrisman SP, Schiff M, Chung SK et al. Implementation of concussion legislation and extent of concussion education for athletes, parents, and coaches in washington state. Am J Sports Med 2014;42:1190-6. 
27. Majerske CW et al. Concussion in sports: Postconcussive activity levels, symptoms, and neurocognitive performance. J Athl Train 2008;43:265-74.

28. Covassin T, Moran R. \& Elbin RJ. Sex differences in reported concussion injury rates and time loss from participation: An update of the national collegiate athletic association injury surveillance program from 2004-2005 through 2008-2009. J Athl Train 2016;51:189-94.

29. Sarmiento K, Rosanne Hoffman R, Dmitrovsky Z et al. A 10-year review of the Centers for Disease Control and Prevention's Heads Up initiatives: Bringing concussion awareness to the forefront. J Saf Res 2014;50:143-7.

30. Lenhart A et al. Teens, social media and technology overview 2015: Smartphones facilitate shifts in communication landscape for teens. Pew Research Center 2015.

31. Ahmed OH, Sullivan SJ, Schneiders AG et al. iSupport: do social networking sites have a role to play in concussion awareness? Disabil Rehabil 2010;32:1877-83.

32. Sullivan SJ et al. 'What's happening?' A content analysis of concussion-related traffic on Twitter. Br J Sports Med 2012;46:258-63.

33. Beakey M, Blake C, Tiernan S et al. Are Irish Sporting Organisations Neglecting the Safety of Their Athletes? A Narrative Review on Concussion Return-to-Play Protocols. Eur J Sport Med 2017;3.

34. Ahmed O, Sullivan SJ, Schneider AG et al. Concussion information online: evaluation of information quality, content and readability of concussion-related websites. $\mathrm{Br} J$ Sport Med 2011-bjsports81620.

35. Mairs K, McNeil H, McLeod J et al. Review Article Online strategies to facilitate health-related knowledge transfer : a systematic search and review. Heal Inf Libr J 
2013;30:261-77.

36. McCrory P. et al. Consensus Statement on Concussion in Sport: The 4th International Conference on Concussion in Sport Held in Zurick, November 2012. Br J Sports Med $2013 ; 47: 250-8$

37. Chinn NR \& Porter P. Concussion reporting behaviours of community college studentathletes and limits of transferring concussion knowledge during the stress of competition. BMJ Open Sport Exerc Med 2016;2:e00118.

38. Tomei KL, Doe C, Prestigiacomo CJ et al. Comparative analysis of state-level concussion legislation and review of current practices in concussion. Neurosurg Focus 2012;33:E11.

39. Newton JD et al. Intention to use sport concussion guidelines among community level coaches and sports trainers. J Sci Med Sport 2013;17:469-73.

40. Baugh CM, Daneshvar DH \& Stern RA. Perceived Coach Support and Concussion Symptom-Reporting: Differences between Freshmen and Non- Freshmen College Football Players. J law Med ethics 2014;314-22.

41. Kroshus E, Baugh CM, Hawrilenko MJ et al. Determinants of Coach Communication About Concussion Safety in US Collegiate Sport. Ann Behav Med 2015;49:532-41.

42. Sarmiento K, Donnell Z. \& Hoffman R. A Scoping Review to Address the Culture of Concussion in Youth and High School Sports*. J Sch Health 2017;87:790-804. 
Table 1. Athlete Demographics and Educational History

\begin{tabular}{|c|c|c|c|c|}
\hline Sport & Male & \multicolumn{2}{|c|}{ Female } & Total (n) \\
\hline Rugby & 866 & \multicolumn{2}{|c|}{231} & 1097 \\
\hline Soccer & 587 & \multicolumn{2}{|c|}{213} & 800 \\
\hline GAA* & 401 & \multicolumn{2}{|c|}{146} & 547 \\
\hline Total & 1854 & \multicolumn{2}{|c|}{590} & 2444 \\
\hline \multicolumn{5}{|c|}{ Concussion Educational Histories of Male and Female Athletes } \\
\hline & & \multicolumn{2}{|c|}{ Total } & \\
\hline & & Male & Female & \\
\hline & & $n=760(41 \%)$ & $n=100(16.9 \%)$ & $\mathrm{n}=860(35.2 \%)$ \\
\hline \multicolumn{5}{|c|}{ Modality } \\
\hline Handou & & $374(49.2 \%)$ & $52(52 \%)$ & $426(49.5 \%)$ \\
\hline Websit & & $188(24.7 \%)$ & 27 (27\%) & $215(25 \%)$ \\
\hline Educati & & 80 (10.5\%) & $5(5 \%)$ & 85 (9.9\%) \\
\hline PC/Vide & & 7 (0.9\%) & - & $7(0.8 \%)$ \\
\hline Present & & 149 (19.6\%) & 15 (15\%) & 164 (19.1\%) \\
\hline Genera & & 388 (51.1\%) & 54 (54\%) & 442 (51.4\%) \\
\hline Interac & ation & $9(1.2 \%)$ & - & $9(1 \%)$ \\
\hline On-fielc & & $11(1.4 \%)$ & - & 11 (1.3\%) \\
\hline Other & & $14(1.8 \%)$ & $4(4 \%)$ & $18(2.1 \%)$ \\
\hline \multicolumn{5}{|c|}{ Messenger } \\
\hline Parents & & 212 (27.9\%) & 35 (35\%) & 247 (28.7\%) \\
\hline Coach & & 225 (29.6\%) & $21(21 \%)$ & $246(28.6 \%)$ \\
\hline Teache & & 109 (14.3\%) & 7 (7\%) & $116(13.5 \%)$ \\
\hline Physio & & 35 (4.6\%) & $5(5 \%)$ & 40 (4.7\%) \\
\hline Guest S & list & 78 (10.3\%) & - & 78 (9.1\%) \\
\hline Fellow & & 47 (6.4\%) & $8(8 \%)$ & 57 (6.6\%) \\
\hline Medica & & 141 (18.6\%) & 12 (12\%) & $153(17.8 \%)$ \\
\hline Profess & layer & $29(3.8 \%)$ & $2(2 \%)$ & 31 (3.6\%) \\
\hline Profess & & $10(1.3 \%)$ & - & $10(1.2 \%)$ \\
\hline Other & & $6(0.8 \%)$ & $3(3 \%)$ & $9(1 \%)$ \\
\hline
\end{tabular}


Table 2. Preferred Methods for Future Concussion Education

\begin{tabular}{|c|c|c|c|}
\hline & \multicolumn{2}{|c|}{ Total } & \multirow{2}{*}{ Total (n) } \\
\hline & Male & Female & \\
\hline \multicolumn{4}{|l|}{ (n) of athletes seeking further education } \\
\hline \multirow[t]{2}{*}{ on SRC } & $1758(94.8 \%)$ & $570(96.6 \%)$ & $2328(95.3 \%)$ \\
\hline & $n=1758$ & $n=570$ & $n=2328$ \\
\hline \multicolumn{4}{|l|}{ Modality } \\
\hline Handout/Poster*† & $132(7.5 \%)$ & $226(39.6 \%)$ & $348(14.9 \%)$ \\
\hline Website/Social media/Mobile App*† & $963(54.8 \%)$ & 239 (41.9\%) & $1202(51.6 \%)$ \\
\hline Educational video*† & $241(13.7 \%)$ & 125 (21.9\%) & $366(15.7 \%)$ \\
\hline PC/Video game*† & $255(14.5 \%)$ & $28(4.9 \%)$ & $283(12.2 \%)$ \\
\hline Presentation*† & $127(7.2 \%)$ & $88(15.4 \%)$ & $215(9.2 \%)$ \\
\hline General conversation*† & $133(7.6 \%)$ & $74(13 \%)$ & 207 (8.9\%) \\
\hline Interactive demonstration*† & $802(45.6 \%)$ & $68(11.9 \%)$ & $870(37.4 \%)$ \\
\hline On-field demonstration*† & $743(42.3 \%)$ & $90(15.8 \%)$ & $833(35.8 \%)$ \\
\hline \multicolumn{4}{|l|}{ Areas of Interest } \\
\hline Signs \& Symptoms & $1465(83.3 \%)$ & $503(88.2 \%)$ & $1968(84.5 \%)$ \\
\hline Incidence & $619(35.2 \%)$ & $194(34 \%)$ & $813(34.9 \%)$ \\
\hline Short-term complications* & 1389 (79\%) & $352(61.8 \%)$ & $1741(74.8 \%)$ \\
\hline Long-term complications* & $662(37.7 \%)$ & $324(56.8 \%)$ & $986(42.4 \%)$ \\
\hline Importance of self-reporting & $498(28.3 \%)$ & $133(23.3 \%)$ & $631(27.1 \%)$ \\
\hline Impact on athletic performance* & $1253(71.3 \%)$ & $188(33 \%)$ & $1461(62.8 \%)$ \\
\hline Impact on academic performance* & $355(20.2 \%)$ & $360(63.2 \%)$ & 715 (30.7\%) \\
\hline Prevention & $1133(64.4 \%)$ & 367 (64.4\%) & $1500(64.4 \%)$ \\
\hline Educational strategies & $124(7.1 \%)$ & $29(5.1 \%)$ & $153(6.6 \%)$ \\
\hline Management/RTP & $1201(68.3 \%)$ & $403(70.7 \%)$ & $1604(68.9 \%)$ \\
\hline Current/future technologies* & $532(30.3 \%)$ & $103(18.1 \%)$ & $635(27.3 \%)$ \\
\hline Legislation & $134(7.6 \%)$ & $49(8.6 \%)$ & $183(7.9 \%)$ \\
\hline Misconceptions/Media* & $359(20.4 \%)$ & $193(33.9 \%)$ & $552(23.7 \%)$ \\
\hline Safety equipment* & 385 (21.9\%) & $287(50.4 \%)$ & $672(28.9 \%)$ \\
\hline High profile cases & $656(37.3 \%)$ & $187(32.8 \%)$ & $843(36.2 \%)$ \\
\hline \multicolumn{4}{|l|}{ Messenger } \\
\hline Parents*† & $137(7.8 \%)$ & $72(12.6 \%)$ & $209(9 \%)$ \\
\hline Coach*† & $784(44.6 \%)$ & $379(66.5 \%)$ & $1163(50 \%)$ \\
\hline Teacher*† & $138(7.8 \%)$ & $73(12.8 \%)$ & $211(9.1 \%)$ \\
\hline Physio* & $86(4.9 \%)$ & $48(8.4 \%)$ & $134(5.8 \%)$ \\
\hline Guest speaker/specialist*† & $664(37.8 \%)$ & $311(54.6 \%)$ & 975 (41.9\%) \\
\hline Fellow rugby player* & $94(5.3 \%)$ & $86(15.1 \%)$ & $180(7.7 \%)$ \\
\hline Medical professional*† & $451(25.7 \%)$ & $399(70 \%)$ & $850(36.5 \%)$ \\
\hline Professional player*† & $1246(70.9 \%)$ & $169(29.6 \%)$ & $1415(60.8 \%)$ \\
\hline Professional coach*† & 1205 (68.5\%) & $197(34.6 \%)$ & $1402(60.2 \%)$ \\
\hline
\end{tabular}




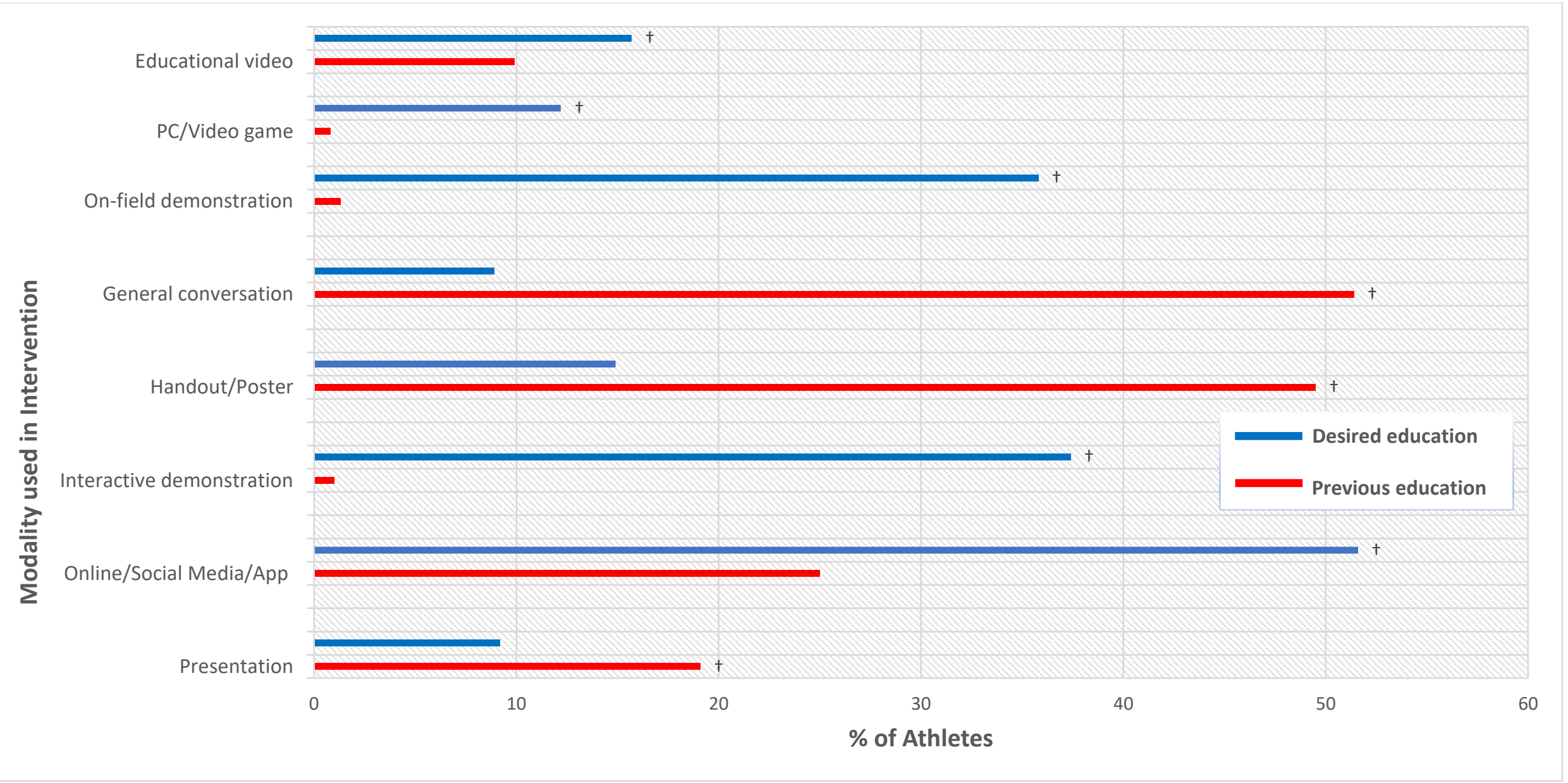

Figure 1. Modality used in Previous Intervention versus Desired Future Modality 


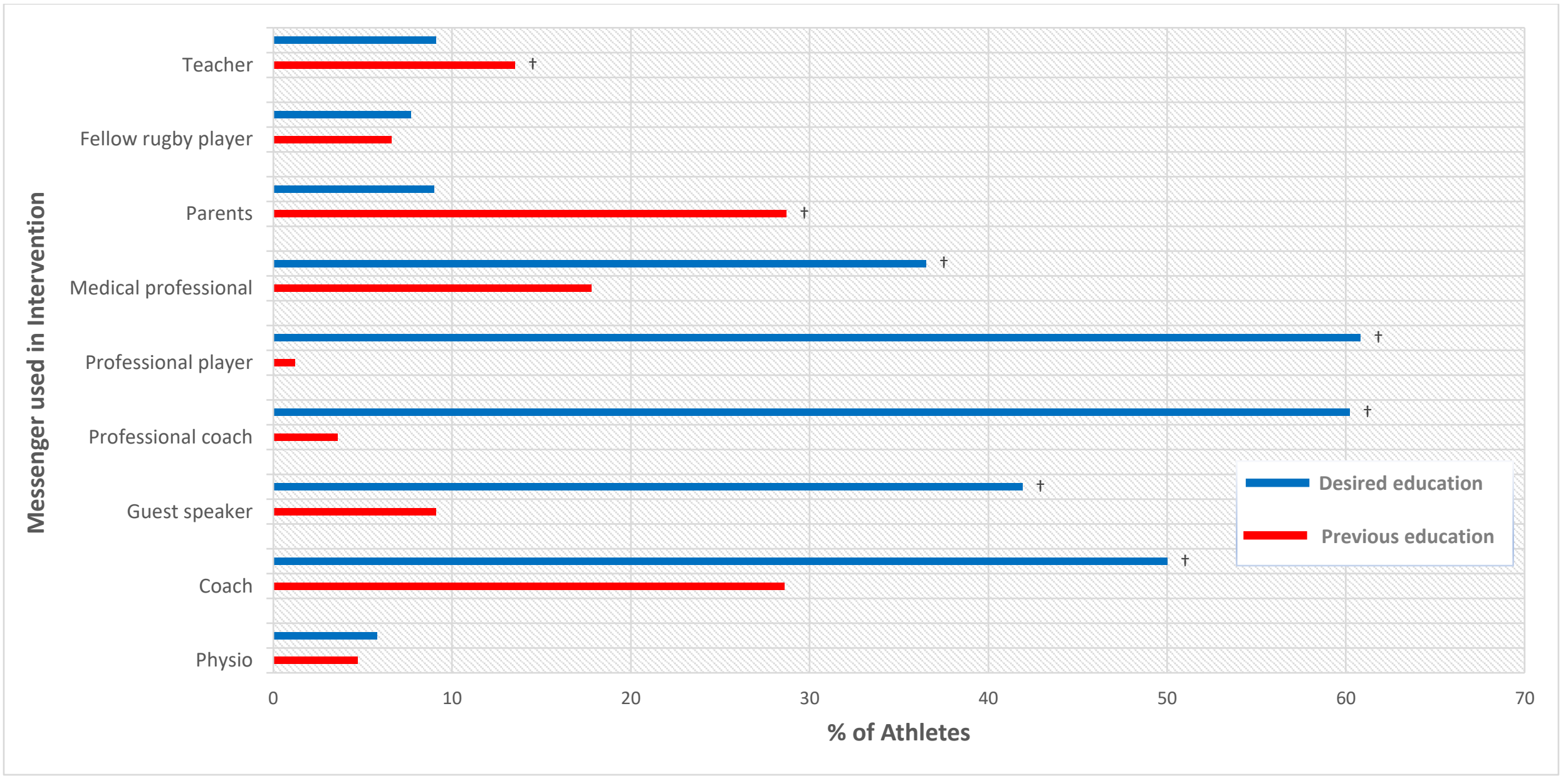

Figure 2. Messenger used in Previous Intervention versus Desired Future Messenger 